\title{
Possibility of sparing umbilicus following hernia repair during abdominoplasty in multiparous obese females
}

\author{
Gamal I El-Habbaa, MD; Mohamed Mahmoud Mohamed Ahmed, MD \\ Department of General Surgery, Benha University, Egypt.
}

\begin{abstract}
Objectives: This study aimed to evaluate the outcome of concomitant umbilical hernial repair and abdominoplasty in multiparous obese females.

Patients \& methods: After application of inclusion criteria, the study included 26 multipareous females with mean age of $30.2 \pm 5.3$ years, number of children of $3.2 \pm 1.1$ child, body mass index (BMI) of $32 \pm 2 \mathrm{~kg} / \mathrm{m}^{2}$ and preoperative waist circumference (WC) was $127.6 \pm 9.9 \mathrm{~cm}$. Operative procedure included open excisional lipectomy with preservation of the umbilical cicatrix with surrounding skin and a leash of blood supply, mesh repair of the umbilical hernia, plication of both rectus sheaths with adjustment of flanks to reduce WC and wound closure after suction drainage. Umbilical preservation outcome, postoperative (PO) WC measurements were taken at one and 6 months after surgery. The frequency of recurrent umbilical hernia was reported at the end of follow-up for 6 months.

Results: Total operative morbidity was $19.2 \%$; 2 patients (7.7\%) had wound infection; one patient (3.8\%) had wound seroma, all had responded to conservative treatment and 2 patients (7.7\%) had wound end dog-ear that was corrected under local anesthesia as outpatient procedure. Twenty patients had well-sited, normally appearing umbilicus with healthy skin and good vascularity with an umbilical preservation success rate of $77 \%$. Six patients had partially impaired vascularity manifested as spots of mild discoloration in 2 patients, superficial skin sloughing of the umbilical edge skin in one patient and umbilical wound infection in one patient. These two patients responded to conservative treatment without wound dehiscence. In 3 patients the umbilicus was slightly caudally shifted. WC estimated at one and 6-m PO showed significant reduction compared to preoperative WC, but was significantly wider compared to WC estimated at $1-m$ PO.

Conclusion: The applied procedure of abdominoplasty with umbilical preservation after repair of umbilical hernia is a safe, effective procedure with good aesthetic results and free of $P O$ hernial recurrence.
\end{abstract}

Key words:Umbilical preservation, abdominoplasty.

\section{Introduction:}

Obesity is a complex, serious medical disorder and a leading public health problem. It contributes to several chronic diseases and is associated with increased mortality rates. ${ }^{1}$ Studies of obesity in populations from Arab Countries in the Middle Eastern Region have been published, indicating that the prevalence of obesity is higher than in most other countries of the world especially in urban areas and among women. ${ }^{2,3}$

The 'nutrition transition' from traditional diets and lifestyles to 'Western' diets (i.e. high in saturated fats, sugar and refined foods), and the combination of reduced levels of physical activity and increased stress were the main factors for increasing prevalence of obesity, particularly in the rapidly growing urban populations ${ }^{4}$. Multiparity and the unofficial vaginal deliveries either or both impose a burden on the abdominal wall musculature with subsequent various types of herniations especially in the umbilical region associated with divarication of recti and lax external oblique aponeurosis resulting in pendulous abdominal belly with disturbed body contour. ${ }^{5}$

Abdominal lipectomy was described in 1890.6 The modern procedure now referred to 
as abdominoplasty has evolved to include lower abdominal lipectomy combined with musculofascial repair. Modification of incision placement and design, as well as variations of musculofascial repair has been reported. ${ }^{7}$

Traditional abdominoplasty techniques for severe deformities include the following procedures: (1) "dermolipectomy" for removal of excess fat and skin, (2) "plication" of the anterior rectus sheath for muscle diastasis, and (3) transposition of the umbilicus to a new location in the abdominal wall skin. Newer techniques developed in the past few years include (1) suction-assisted lipectomy, (2) high lateral tension closure with fascial suspension, and (3) external oblique fascial advancement to create a smaller waistline. Some of these techniques may have drawbacks, and the newer maneuvers, particularly the external oblique muscle advancement, can be technically challenging. 8

This study aimed to evaluate the outcome of concomitant umbilical hernial repair and abdominoplasty in multiparous obese females.

\section{Patients and methods:}

The present prospective study was conducted at General Surgery Department, Benha University Hospital, since July 2006 till March 2009 to allow 6 months for the last operated case. After approval of the study protocol by the Local Ethical Committee and obtaining written fully informed patients' consent 26 female multiparous patients were enrolled in the study. This surgery is indicated in patients how had umbilical hernia with abdominal laxness, extensive striaes, rectus muscle diastases, and localized lipodystrophy. Exclusion criteria included presence of cardiac or respiratory diseases, intra-abdominal pathologies requiring laprotomy, uterine prolapse or other problems and hematological disorders. Patients had abdominal scar for previous surgical interference, incisional hernias, recurrent umbilical or para-umblical hernia were excluded of the study.

The predetermined incision was drawn so as to include the umbilicus with about $3 \mathrm{~cm}$ supraumblical skin, Figure(1). The supraumbilical incision was first done and an epigastric tunnel was undermined to the xiphoid process with preservation of the lateral superior flank area and sparing the neurovascular bundles that support the entire flap, Figure(2). The patient was put in the Fowler position and the flap was pulled down to try to reach the previously delineated inferior line (inferior wound edge) so as to estimate the extent to which the flap resection can be done en bloc without problems and defect closure without tension. The inferior flap was dissected down depending on the laxness and elasticity of the skin, however, for all patients lower flap was dissected to approximately $7-8 \mathrm{~cm}$ from the labia major. After confirming the safety of the pull-down approach, the entire flap was raised en bloc from the abdominal wall and excised, Figure $(3,4)$. The patient was again placed in the horizontal position and the umbilical hernia was dissected and the edges of the defect edges were identified, Figure(5); then the hernial sac with its contents and peritoneal reflections were reduced intra-abdominally and muscular defect edges were fully dissected. Then a piece of proline mesh was introduced into the defect and spread as a tension-free underlay submuscular peri-peritoneal graft and was anchored to anterior abdominal wall away from the defect, Figure(6,7). Both recti were plicated in the midline from the xiphoid appendix to the suprapubic area using proline suture; the suture line was started from the epigastric area down to and sparing the umbilicus stack in a medium-to-firm fixation, Figure(8). Muscle plication was designed so as to achieve a waist improvement of approximately $8 \mathrm{~cm}$. The umbilical stack was trimmed to an elliptical shape; with a No. 11 blade, a longitudinal incision approximately $2 \mathrm{~cm}$ long was made in the remnant of the cephalic flap. The patient was put in a semi-Fowler position and the flap was extended to the pubis area. A 2-0 nylon stitch was placed to support the flap, and, using Allis forceps, the umbilicus stack was grasped and projected to the abdominal wall and the umbilicus was fixed to the edges of the skin incision. Nonabsorbable 4-0 stitches were placed in the subcutaneous level, the scarpa fascia border was sutured together with the subcutaneous flap to protect the lymphatic vessels and musculocutaneous nerves. Then, two suction drains were inserted in each flank and subcutaneous and intradermic sutures were made with vicryl 3-0. Postoperatively, support 
corset was placed on the patient to reinforce and maintain pressure on the abdomen wall. Postoperative analgesia, broad-spectrum antibiotics, fluid therapy and low-molecular weight heparin were injected subcutaneously for 7 days. Patients were instructed to move legs beginning immediately after the surgery with concomitant leg massage to prevent deep venous thromboembolism (DVT). On the next day, the patient was allowed to walk slowly in a hunched position to avoid stretching the abdomen. Once patients were tolerant for selfdependent home stay, they were discharged. The drains were maintained till dry and patients were instructed to attend the General Surgery Outpatient Clinic for drain removal. Data collection:

Preoperative evaluation included age, parity, body anthropometric measures including weight, height, waist circumference were measured and body mass index (BMI; calculated as the weight in kilograms divided by the height in meters squared) was determined, overweight was a BMI of 25 to $<30 \mathrm{~kg} / \mathrm{m}^{2}$ and obesity was a BMI of $\cdot 30$ $\mathrm{kg} / \mathrm{m}^{2}$ or more. ${ }^{9}$

Operative data included duration of surgery, intraoperative amount of blood loss, frequency and number of transfused blood bags. Immediate postoperative data included duration of wound drainage, duration of hospital stay and types and frequency of postoperative complications including wound seroma or hematoma, wound infection, DVT and lines of management provided. Operative related morbidity and mortality were recorded.

\section{Surgical Outcome Parameters}

1. Umbilical preservation outcome was evaluated prior to discharge in the following items:

a. Umbilical appearance graded as normal or distorted

b. Umbilical site in relation to the distance from symphysis pubis to xiphosternum

c. Umbilical skin vascularity; whether discolored, sloughed or infected.
2. Considering waist circumference measurements as a measure for abdominoplasty outcome, WC was estimated preoperatively, at 1 and 6 months after surgery.

3. The frequency of recurrent umbilical hernia was reported at the end of follow-up for 6 months.

\section{Case Presentation}

\section{Case 1:}

Figure(1a): Showing a pendulous abdomen in an obese female with bulging waist and umbilical hernia. Demarcation lines for the proposed incision for abdominoplasty were shown and included the umbilicus and about $3 \mathrm{~cm}$ supraumblical skin.

Figure(1b): Showed the superior wound edge including the umbilicus with the supraumblical skin.

Figure(1c): Showed both cephalic and caudal abdominal wall flaps were completely dissected off the underlying abdominal muscles. Umbilicus (U) with its leash of blood supply and surrounding skin were preserved. Umbilical hernia $(\mathrm{H})$ was bulging.

Figure(1d): Showed the excised abdominal wall specimen having a cephalid notch of the excised umbilicus and surrounding skin.

Figure(1e): Showed the umbilical hernia $(\mathrm{H})$ was dissected and reduced intraabdominally and the edges (E) of the anterior abdominal defect were identified. The umbilicus (U) with its leash of blood supply was preserved.

Figure(1f): Showed the 4 angles of the hernial defect were suspended by proline stitches and underlay piece of proline mesh (M) was introduced into the defect for abdominal wall enforcement.

Figure(1g) Showed that the hernial repair was completed.

Figure(1h) Showed plication of the muscle aponeurosis diastases, fixing the umbilicus stack with preservation of the lateral neurovascular bundles. 


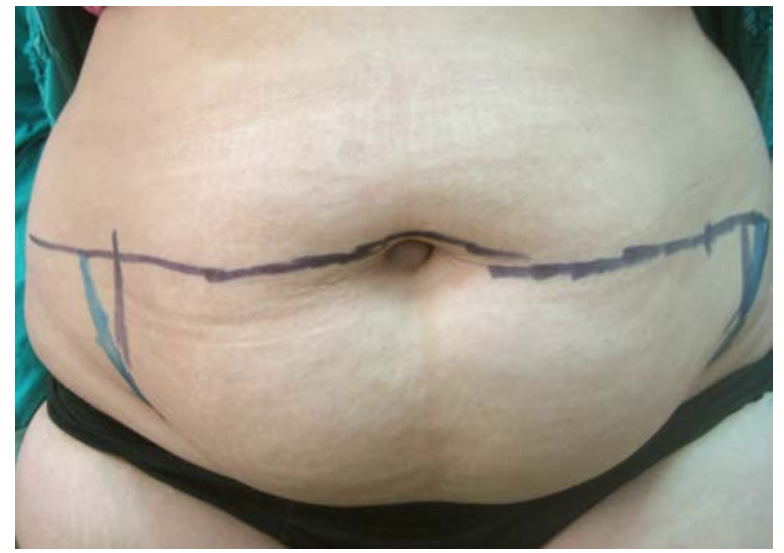

(A)

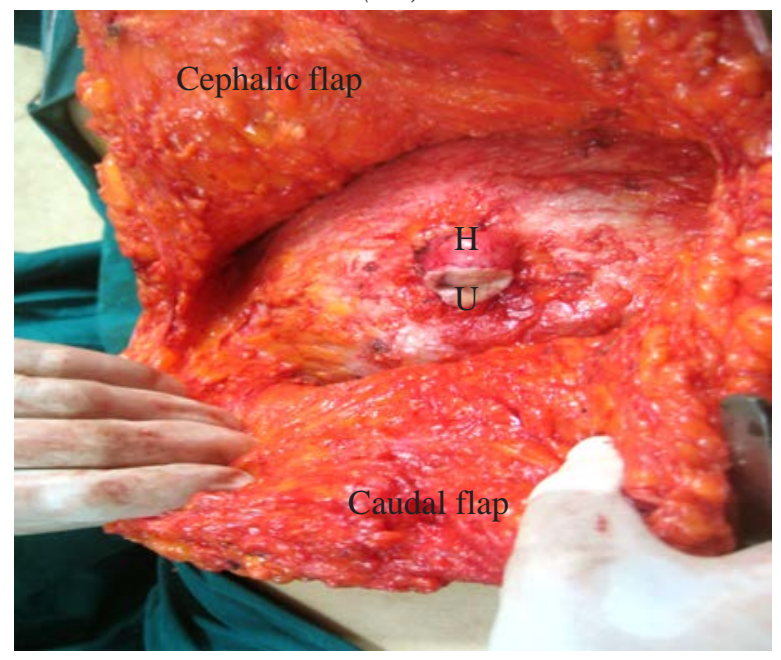

(C)

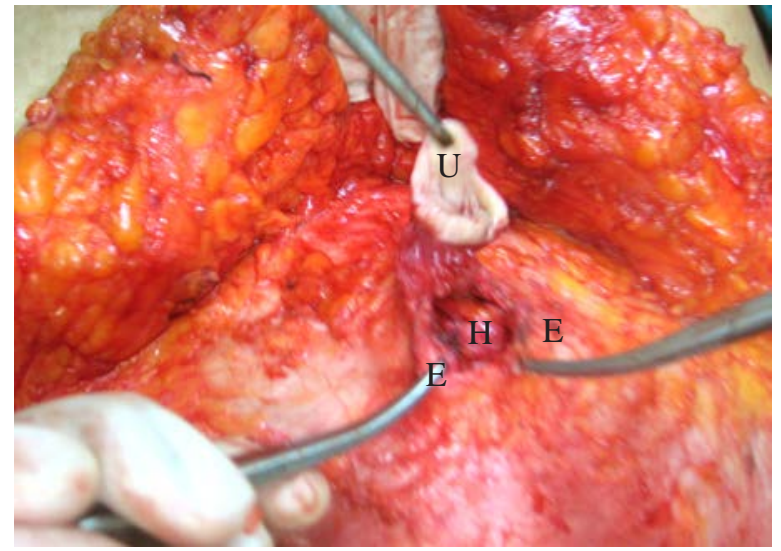

(E)

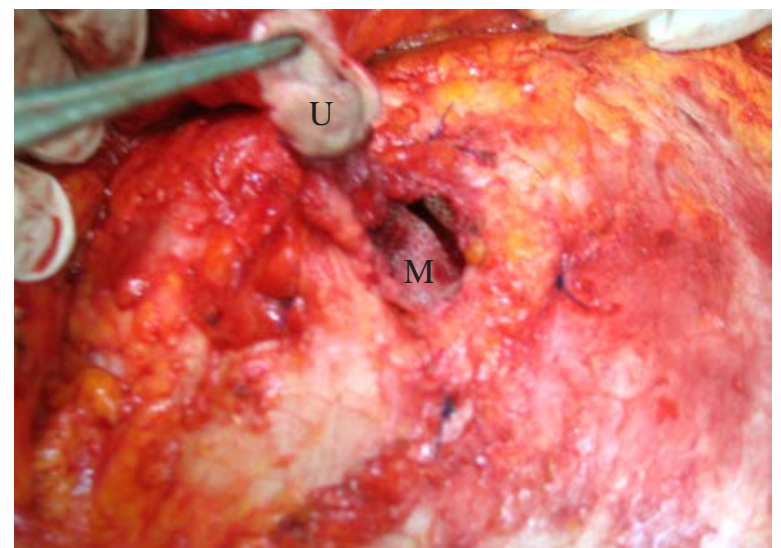

$(G)$

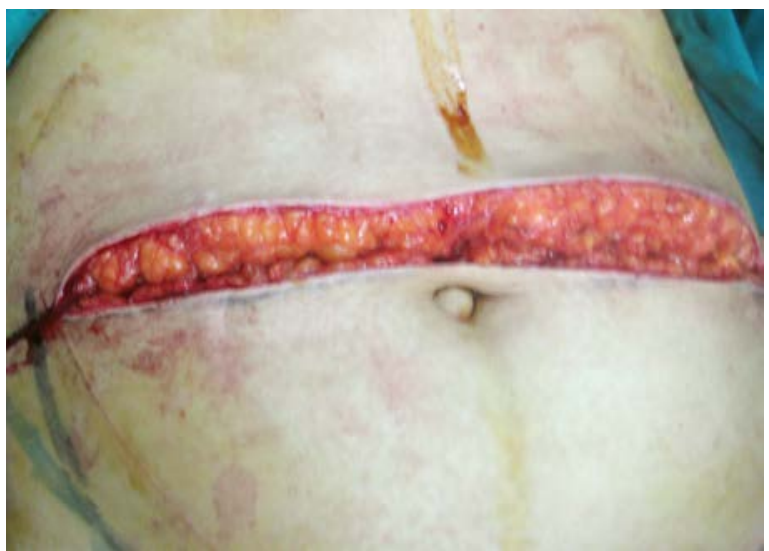

(B)

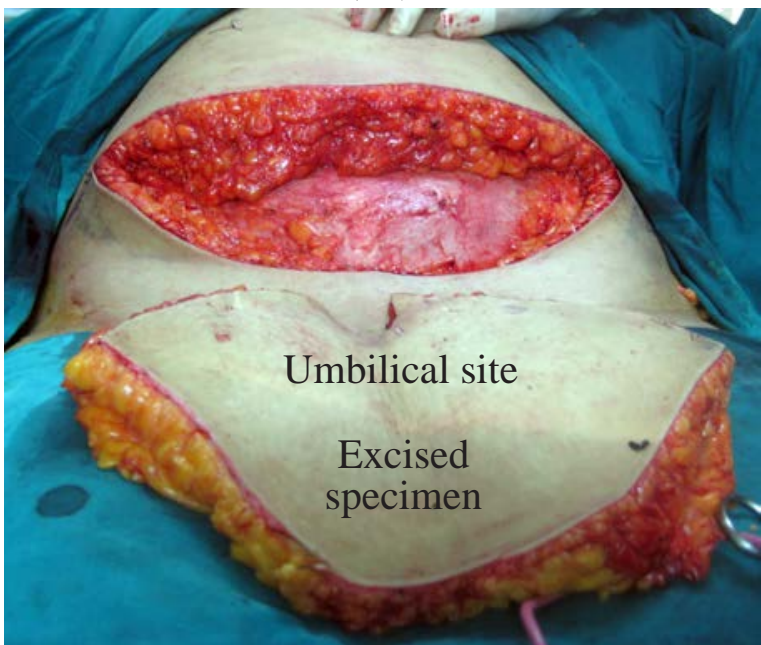

(D)

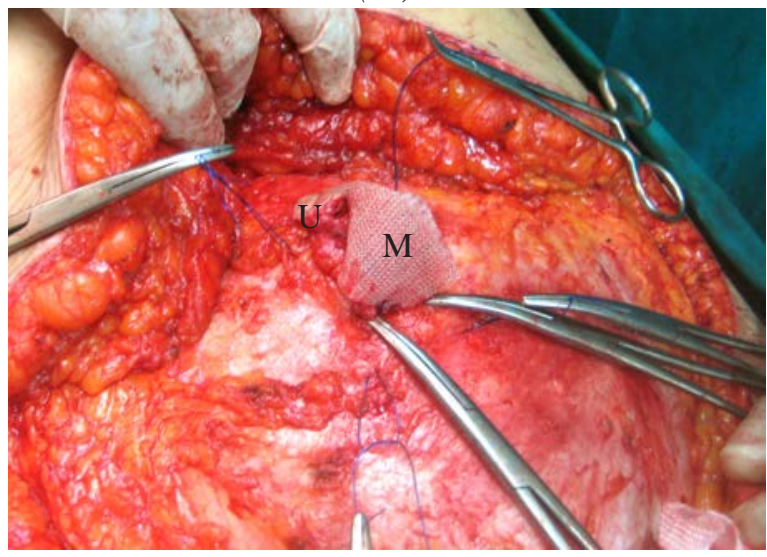

$(F)$

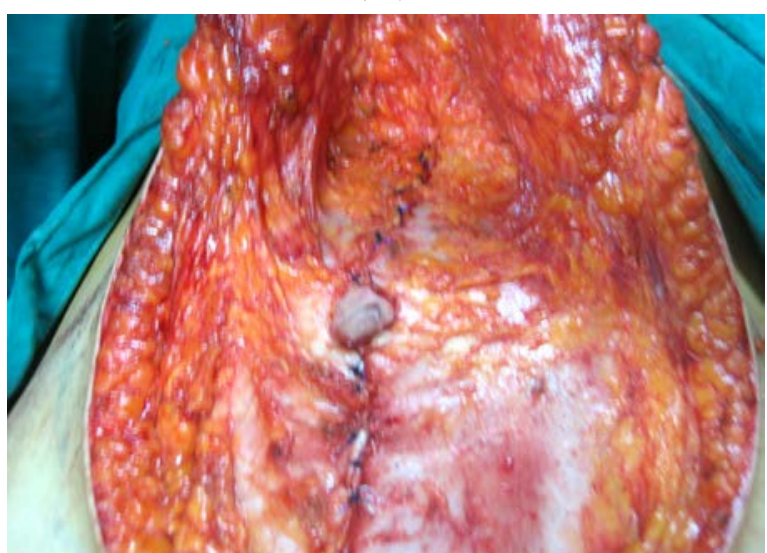

(H)

Figure (1) 


\section{Case 2:}

Figure(2 a,b): Showing a pendulous abdomen with abdominal belly encroaching on the vulval region and upper thigh. Patient had supraumbilical hernia. Demarcation lines for the proposed incision for abdominoplasty were shown and included the umbilicus and about $3 \mathrm{~cm}$ supraumblical skin.

Figure(2c): Showed both cephalic and caudal abdominal wall flaps were completely dissected off the underlying abdominal muscles. Umbilicus (U) and surrounding skin were preserved. Umbilical hernia $(\mathrm{H})$ was bulging.

Figure(2d): Showed the umbilical hernia (H) was dissected and reduced intra-

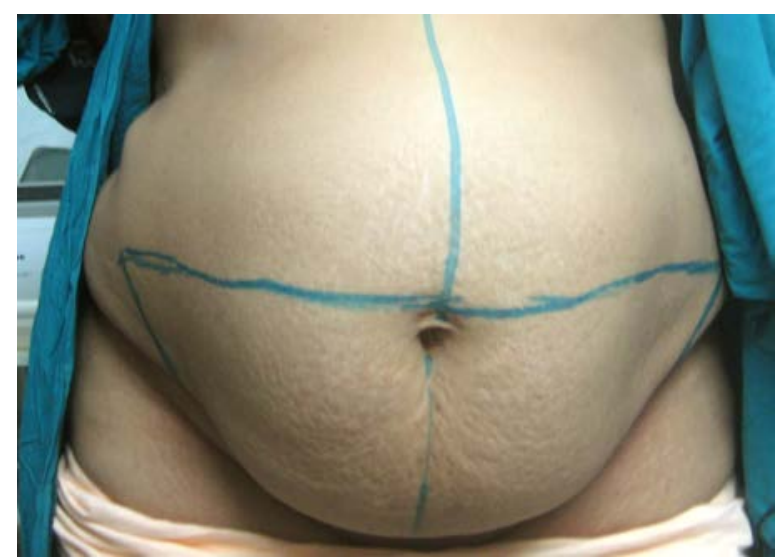

(A)

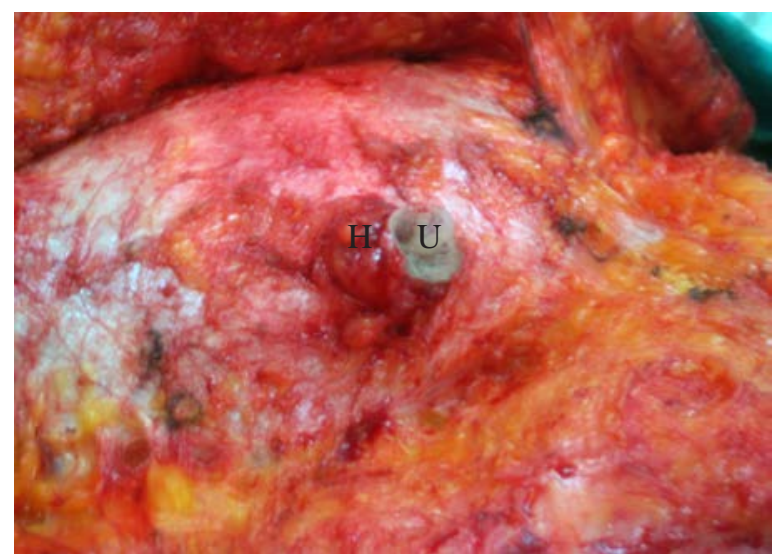

(C)

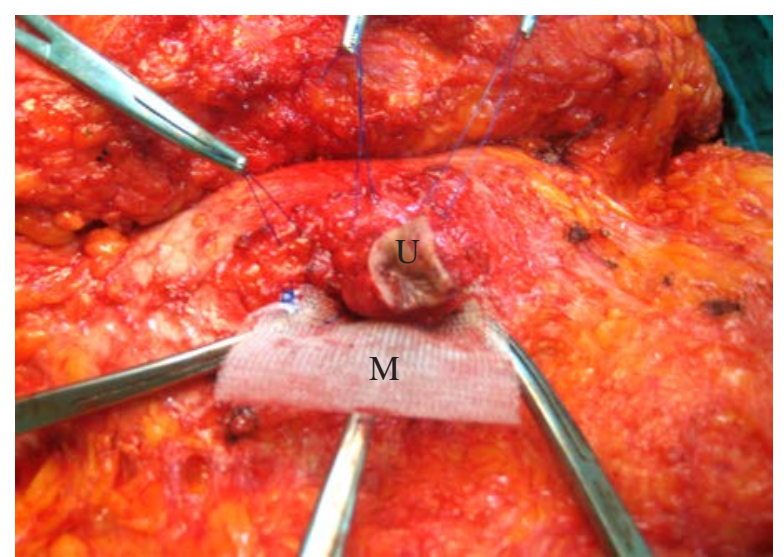

(E) abdominally and the edges (E) of the anterior abdominal defect were identified. The umbilicus (U) with its leash of blood supply was preserved.

Figure(2e,f): Showed the angles of the hernial defect were suspended by proline stitches and underlay piece of proline mesh (M) was introduced into the defect for abdominal wall enforcement.

Figure(2g): Showed that the hernial repair was completed.

Figure(2h): Showing immediate postoperative abdominal appearance after wound closure and disappearance of the abdominal belly.

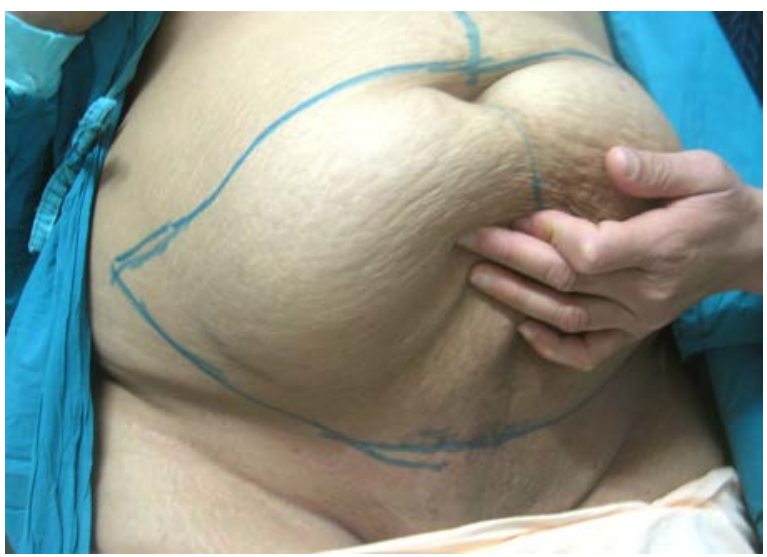

(B)

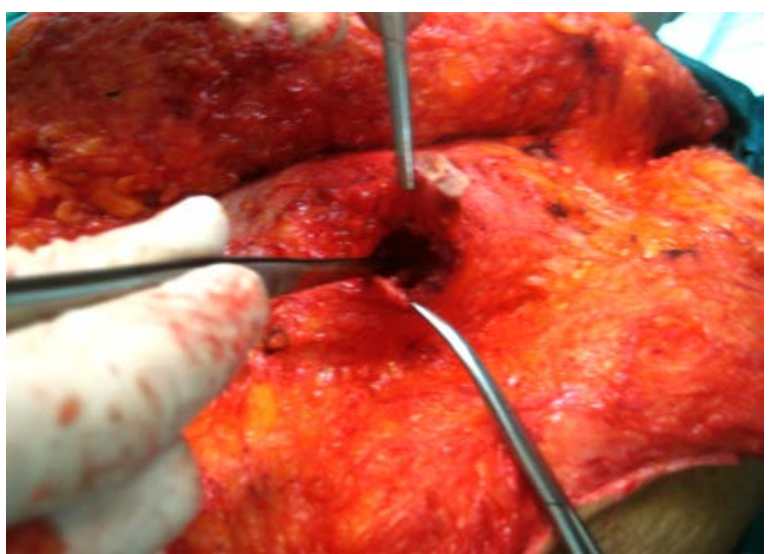

(D)

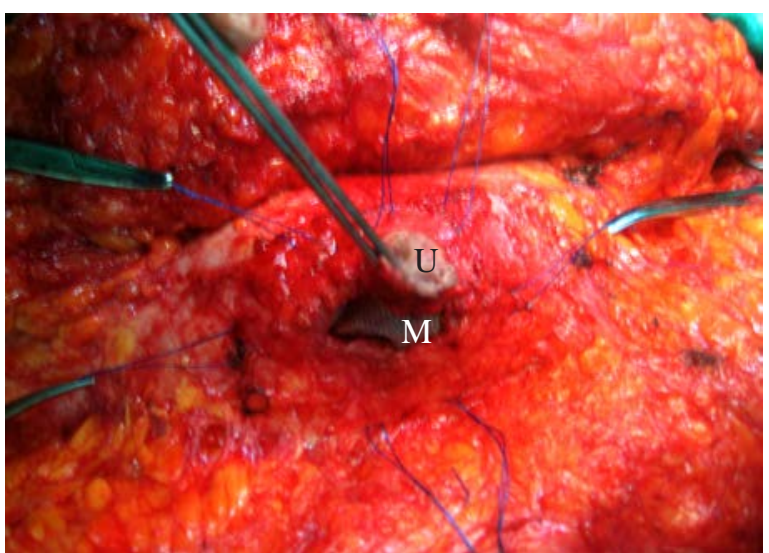

$(F)$ 


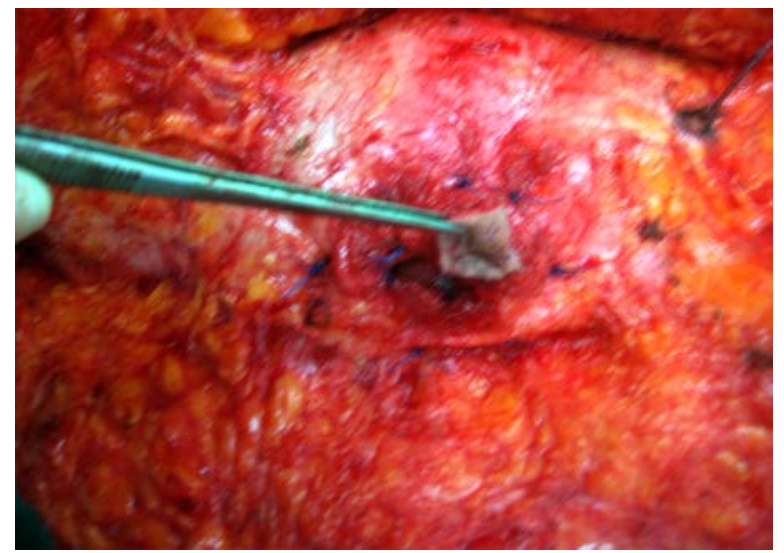

$(G)$

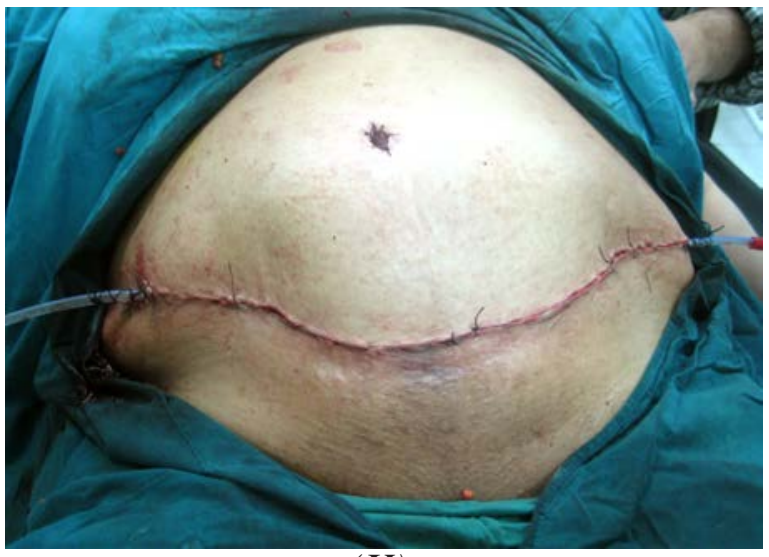

(H)

Figure (2)

\section{Results:}

The study included 26 multipareous females with mean age of $30.2 \pm 5.3$; range: $22-41$ years and mean number of children of $3.2 \pm 1.1$; range: 2-6 children. Only 5 patients were overweight with BMI $<30 \mathrm{~kg} / \mathrm{m}^{2}$, while 21 patients were obese with BMI $>30 \mathrm{~kg} / \mathrm{m}^{2}$ and a mean total BMI of $32 \pm 2$; range: $27-34.8 \mathrm{~kg} / \mathrm{m}^{2}$. Mean patients' preoperative WC was 127.6 \pm 9.9 ; range: $110-152 \mathrm{~cm}$, Table(1).

All patients passed smooth intraoperative course with mean duration of surgery of 142.5 \pm 34 ; range: $100-210$ minutes and mean blood loss of $185 \pm 61.4$; range: $100-300 \mathrm{cc}$ that did not necessitate blood transfusion and fluid replacement with lactated Ringer' solution was sufficient to maintain hemodynamic stability, Table(2).

Mean hospital stay was continued for a mean duration of 4.3 \pm 0.9 ; range: $3-6$ days; mean duration of wound drainage was 13.2 \pm 3.9 ; range: 8-20 days, $\mathbf{T a b l e}(2)$. Three patients $(11.5 \%)$ attended the hospital prior to time of first PO visit; 2 patients $(7.7 \%)$ had signs of wound infection with redness and tenderness of wound edge and concomitant mild elevation of body temperature. Both patients were re-admitted and responded to conservative treatment without active interference. One patient $(3.8 \%)$ had wound seroma but showed no signs of infection and was not admitted and was given broad specterum antibiotics and ant-inflammatory anti-edematous therapy. Next week the seroma subsided without other complications, Table(3).
Throughout immediate PO period no cases developed DVT or embolism and no mortality was reported. After a mean follow-up period of 14.3 \pm 5.4 ; range: $6-30$ months, no recurrent umbilical hernia was recorded. Only 2 patients (7.7\%) had wound end dog-ear and were repaired under local anesthesia as outpatient procedure and completely disappeared in one and significantly reduced in the other patient. Thus, the total operative morbidity was $19.2 \%$, Table(3).

As regards umbilical preservation as an outcome, 20 patients had well-sited, normally appearing umbilicus with healthy skin and good vascularity with an umbilical preservation success rate of $77 \%$. Six patients had partially impaired vascularity manifested as spots of mild discoloration in 2 patients, superficial skin sloughing of the umbilical edge skin in one patient and umbilical wound infection in one patient. These two patients responded to conservative treatment consisting of local frequent dressings and no wound dehiscence was reported, but unfortunately umbilical appearance was to some extent distorted. In 3 patients the umbilicus was slightly caudally shifted, Table(4).

Waist circumference estimated at 1-m after surgery showed significant reduction compared to preoperative WC. Moreover, WC estimated at 6-months after surgery was still significantly reduced compared to preoperative $\mathrm{WC}$, despite being significantly wider than that measured at 1-m after surgery, Table(5), Figure(4,5). 
Table (1): Patients' preoperative data.

\begin{tabular}{|c|c|c|c|}
\hline \multicolumn{3}{|l|}{ Age (years) } & $30.2 \pm 5.3(22-41)$ \\
\hline \multicolumn{3}{|l|}{ Parity (children) } & $3.2 \pm 1.1(2-6)$ \\
\hline \multirow{6}{*}{$\begin{array}{l}\text { Anthropometric } \\
\text { data }\end{array}$} & \multicolumn{2}{|l|}{ Weight (kg) } & $88.2 \pm 5.2(77-95)$ \\
\hline & \multicolumn{2}{|l|}{ Height $(\mathrm{cm})$} & $166.1 \pm 4.5(155-171)$ \\
\hline & \multirow[t]{3}{*}{$\mathrm{BMI}\left(\mathrm{kg} / \mathrm{m}^{2}\right)$} & Mean \pm SD & $32 \pm 2(27-34.8)$ \\
\hline & & $\mathrm{BM} \mathrm{M}<30$ & $5(19.2 \%)$ \\
\hline & & $\mathrm{BMI}>30$ & $21(80.8 \%)$ \\
\hline & \multicolumn{2}{|c|}{ Waist circumference $(\mathrm{cm})$} & $127.6 \pm 9.9(110-152)$ \\
\hline
\end{tabular}

Data are presented as mean $\pm S D$ \& numbers; ranges \& percentages are in parenthesis

Table (2): Patients' operative and hospital stay data.

\begin{tabular}{|l|c|c|}
\hline & Mean \pm SD & Range \\
\hline Duration of surgery (min) & $142.5 \pm 34$ & $100-210$ \\
\hline Amount of blood loss (cc) & $185 \pm 61.4$ & $100-300$ \\
\hline Duration of hospital stay (days) & $4.3 \pm 0.9$ & $3-6$ \\
\hline Duration of wound drainage (days) & $13.2 \pm 3.9$ & $8-20$ \\
\hline
\end{tabular}

Table (3): Postoperative data.

\begin{tabular}{|c|c|c|c|c|}
\hline \multicolumn{2}{|l|}{ Time } & & Data & Management \\
\hline \multirow{6}{*}{\multicolumn{2}{|c|}{ Early }} & Wound infection & $2(7.7 \%)$ & \multirow{2}{*}{$\begin{array}{c}\text { Responded to } \\
\text { conservative therapy }\end{array}$} \\
\hline & & Seroma & $1(3.8 \%)$ & \\
\hline & & DVT & 0 & \\
\hline & & Early hernial recurrence & 0 & \\
\hline & & Wound end dog-ear & $2(7.7 \%)$ & Repaired \\
\hline & & Total operative morbidity & $5(19.2 \%)$ & \\
\hline Follow-up & \multicolumn{2}{|c|}{ Duration (months) } & $14.3 \pm 5.4(6-30)$ & \\
\hline & \multirow[t]{2}{*}{ Events } & Hernial recurrence & 0 & \\
\hline & & Mortality & 0 & \\
\hline
\end{tabular}

Table (4): Umbilical vascularity preservation data.

\begin{tabular}{|l|c|c|}
\hline Items & & Number (\%) \\
\hline \multirow{2}{*}{ Umbilical appearance } & Normal & $23(88.5 \%)$ \\
\cline { 2 - 3 } & Distorted & $3(11.5 \%)$ \\
\hline \multirow{3}{*}{ Umbilical site } & Normal & $22(84.6 \%)$ \\
\cline { 2 - 3 } & Shifted upwards & $1(3.8 \%)$ \\
\cline { 2 - 3 } & Shifted downwards & $3(11.5 \%)$ \\
\hline \multirow{3}{*}{ Umbilical skin vascularity } & Healthy with good vascularity & $22(84.6 \%)$ \\
\cline { 2 - 3 } & Healthy with partially impaired vascularity & $2(7.7 \%)$ \\
\cline { 2 - 3 } & Superficial sloughing & $1(3.8 \%)$ \\
\cline { 2 - 3 } & Infection & $1(3.8 \%)$ \\
\hline
\end{tabular}


Table (5): Waist circumference change data.

\begin{tabular}{|c|c|c|c|c|}
\hline & Mean \pm SD $(\mathrm{cm})$ & Range $(\mathrm{cm})$ & \multicolumn{2}{|c|}{ Statistical analysis } \\
\cline { 3 - 5 } & & & $\mathrm{Z}$ & $\mathrm{P}$ \\
\hline Preoperative & $127.6 \pm 9.9$ & $110-152$ & & \\
\hline At 1-m PO & $115 \pm 9.6$ & $99-140$ & 4.491 & $\mathrm{P} 1<0.001$ \\
\hline At 6-m PO & $115.8 \pm 9.5$ & $100-140$ & 4.481 & $\mathrm{P} 1<0.001$ \\
& & & 3.179 & $\mathrm{P} 2=0.001$ \\
\hline
\end{tabular}

$P 1$ : significance versus preoperative circumference

P2: significance versus circumference estimated at 1-m PO

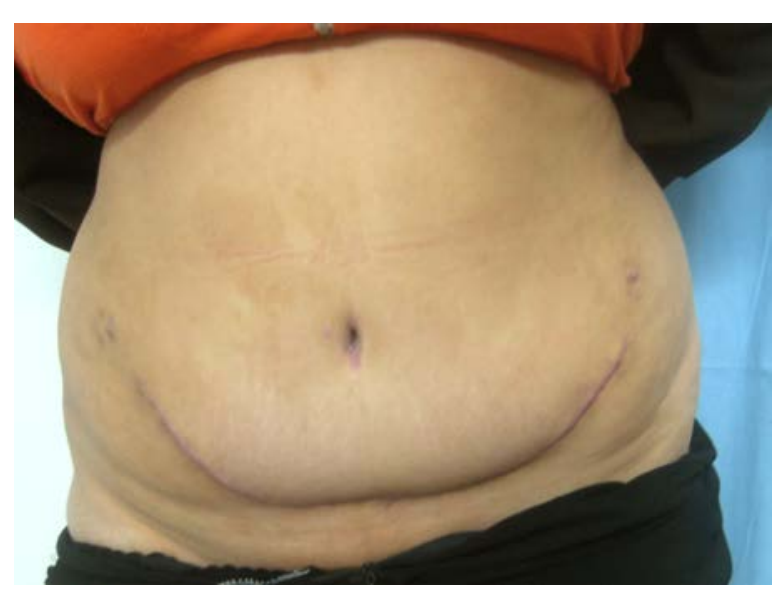

Figure(3)

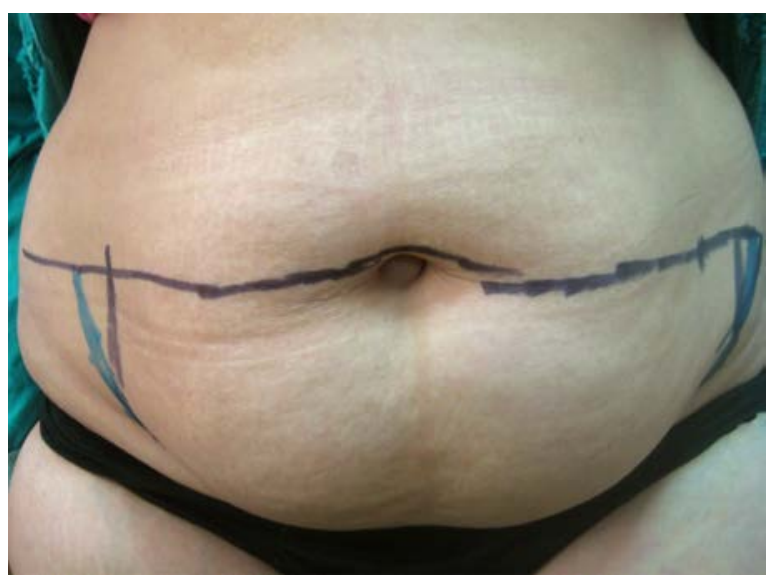

Figure(1a)

Figure (3): showing postoperative abdominal appearance after complete wound healing (at 6m PO); in comparison to preoperative one Figure( 1 a) waist reduction was evident with disappearance of the redundant abdominal belly.

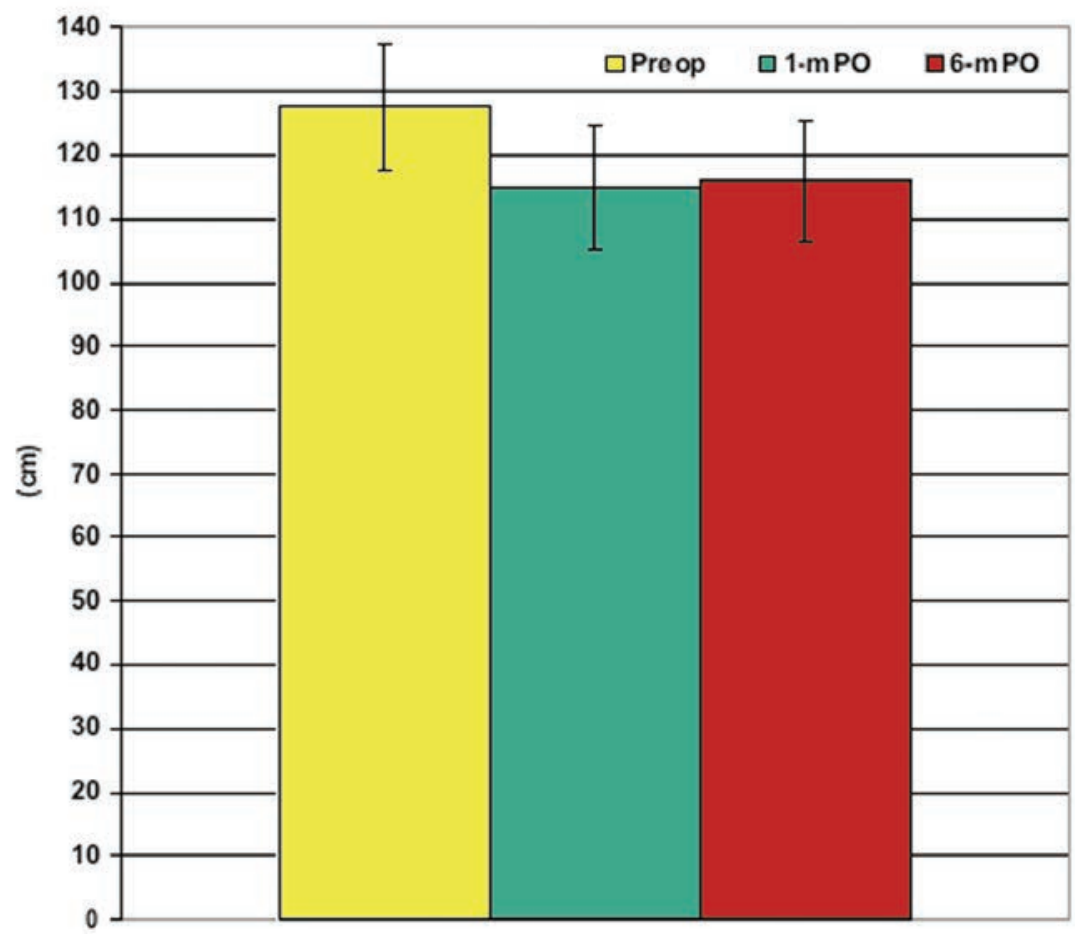

Figure (4): Mean ( $\pm S D$ ) waist circumference estimated throughout the study. 


\section{Discussion:}

The present study presents a trial for concomitant umbilical hernial repair and abdominoplasty in the same setting for 26 multipara with high BMI. Only 4 patients had mild resolving umbilical vascular problems and 20 patients had normally appearing good vascular umbilicus situated at normal distance between symphysis pubis and xiphisternum. Such maintained umbilical vascularity was dependent on preservation of the leash of blood supply for the umbilicus throughout the procedure with dissection of the umbilical hernia away from it so as neither to include that leash with the peritoneal reflection to be dissected, excised and sutured nor with the stitches applied for fixation of the applied mesh repair.

Moreover, the inclusion of about $1 \mathrm{~cm}$ of skin all-around the umbilicus allowed fixing it at the new site without applications of stitches through the umbilical skin thus preventing distortion, eversion or disappearance of the umbilical dimple. This allowed normally appearing anterior abdominal wall.

The conducted abdominoplasty was dependent on pull-down technique so as to use the skin of the upper abdominal wall that normally was not included in the redundant belly and had minimal stria marking thus helped rejuvenating the abdominal appearance.

Considering waist measurement reduction as outcome of abdominoplasty, waist circumference estimated prior to discharge was significantly reduced compared to preoperative measurement, this could be attributed both to the excision of excess redundant skin en-block with subcutaneous fat, extending the excision to the flanks and more importantly to the midline rectus sheath plication with adjustment of the extent of plication so as to reduce the waist circumference.

The applied procedure did not rely on external oblique aponeurosis plication to avoid dissymmetry and muscle traction wich mostly induced postoperative limitation in abdominal relaxation during inspiration and may limit proper ventilation. Such policy with minimization of muscle stitching and avoidance of extensive caudal dissection allowed early postoperative ambulation with its consequent benefits for avoidance of DVT, early resumption of oral intake and shortening of hospital stay.

Closure of the resultant dead space with multiple stitches and preservation of lymphatics minimized the seroma formation, also, the bilateral suction drainage for sufficient time aided to minimize seroma despite the wide dissection area.

As regards the outcome of applied procedure for abdominoplasty, the obtained results coincided with Uebel ${ }^{10}$ who applied similar pull-down technique, however, depended on fat debulking of the reminant flaps and this may affect the skin vascularity or endanger lymphatics with subsequent seroma and nonpitting skin edema. The dependence on vascularized umbilicus obviated the need for further maneuvers to provide the umbilical cicatrix with blood supply and provided natural appearance of the umbilical concavity, thus superceded the results of Rozen \& Redett ${ }^{11}$ who created two dermal flaps through deepithelialization and incision of skin in the neoumbilical position in the midline, then both flaps were sutured down to the abdominal fascia, thereby creating a natural periumbilical concavity.

Also, the applied procedure was superior to that applied by Bozola ${ }^{12}$ who depended on preliminary liposuction followed by removal of all the infraumbilical skin and a subcutaneous segment after detachment of the umbilicus aponeurotic implantation, then conducted a median tunnel from the umbilicus to the xiphoid process with plication of the rectus muscle aponeurosis from the pubis to the xiphoid process, then the umbilicus was transposed. Despite similarity in procedure, the current study did not rely on liposuction with its inherent complication especially the blind injuries of blood and lymphatic vessels and the dissimilarity of areas of suction. ${ }^{13}$ Furthermore, Bozola ${ }^{12}$ detached the umbilical stump and re-attach it thus the umbilicus was dependent for blood supply on neovascularization as a free graft, but through the current study blood supply was preserved throughout the procedure and thus the healing of the umbilical wound and survival of the umbilicus depended on its own blood supply. 
As regards umbilical hernia repair with umbilical conservation, the obtained results were in line with Bruner et al. ${ }^{14}$ who tried abdominoplasty with umbilical hernia and reported that historical concerns regarding circulatory compromise of the umbilicus as a result of simultaneous repair of such hernias with abdominoplasty were found to be unwarranted and none of the patients have experienced a recurrence of their umbilical hernia or necrosis of their umbilicus with improved overall aesthetics of the umbilicus.

Throughout the study period the total operative morbidity was $19.2 \%$, but all complications were minor and resolvable; such figure goes in hand with Heller et al. ${ }^{15}$ who reported a complication rate of $17 \%$ after modified low transverse abdominoplasty and with Batchvarova et al. ${ }^{16}$ who reported variant minor complication in 10 of 52 patients after abdominoplasty with plication of the posterior rectus fascia and enforcement using a resorbable mesh.

In conclusion; the applied procedure of abdominoplasty with umbilical preservation after repair of umbilical hernia is a safe, effective procedure with good aesthetic results and free of PO hernial recurrence.

\section{References:}

1- Jakicic JM, Otto AD: Physical activity considerations for the treatment and prevention of obesity. American Journal of Clinical Nutrition 2005; 82(1): 226-229.

2- Abdul-Rahim HF, Abu-Rmeileh NME, Husseini A, Holmboe-Ottesen G, Jervell J, Bjertness E: Obesity and selected comorbidities in urban Arabian populations. Int J Obesity 2001; 25: 1736-1740.

3- Al-Nouzha MM, Al-Mazrou YY, AlMaatouq MA, Arafaa MR, Khalil MZ, Khan NB, et al: Obesity in Saudi Arabia. Saudi Med J 2005; 26(5): 824-829.

4- Amarasinghe A, D'Souza G, Brown C, Oh $\mathrm{H}$, Borisova $\mathrm{T}$ : The influence of socioeconomic and environmental determinants on health and obesity: A West Virginia case study. Int J Environ Res Public Health 2009; 6(8): 2271-2287.
5- Sozer SO, Agullo FJ, Santillan AA, Wolf C: Decision making in abdominoplasty. Aesthetic Plast Surg 2007; 31(2):117-127.

6- Elbaz JS, Flageul G: Plastic surgery of the abdomen. New York; Masson (Publisher); 1979; p. 42.

7- Vila-Rovira R: Lipoabdominoplasty. Clin Plast Surg 2008; 35(1): 95-105.

8- Murshid M, Khalid KN, Shakir A, Bener A: Abdominoplasty in obese and in morbidly obese patients. J Plast Reconstr Aesthet Surg 2009; Epub ahead of print.

9- Obesity Education Initiative Expert Panel: Clinical guidelines on the identification, evaluation and treatment of overweight and obesity in adults-the evidence report. Obes Res 1998; 6(2 Suppl): 51-209.

10-Uebel CO: Lipoabdominoplasty: Revisiting the superior pull-down abdominal flap and new approaches. Aesthetic Plast Surg 2009; 33(3): 366-376.

11-Rozen SM, Redett R: The two-dermal-flap umbilical transposition: A natural and aesthetic umbilicus after abdominoplasty. Plast Reconstr Surg 2007; 119(7): 22552262.

12-Bozola AR: Abdominoplasty: Same classification and a new treatment concept 20 years later. Aesthetic Plast Surg 2009; Epub ahead of print.

13-Kolker AR: Improving esthetics and safety in abdominoplasty with broad lateral subcostal perforator preservation and contouring with liposuction. Ann Plast Surg 2008; 60(5): 491-497.

14-Bruner TW, Salazar-Reyes H, Friedman JD: Umbilical hernia repair in conjunction with abdominplasty: A surgical technique to maintain umbilical blood supply. Aesthet Surg J 2009; 29(4): 333-334.

15-Heller JB, Teng E, Knoll BI, Persing J: Outcome analysis of combined lipoabdominoplasty versus conventional abdominoplasty. Plast Reconstr Surg 2008; 121(5): 1821-1829.

16-Batchvarova Z, Leymarie N, Lepage C, Leyder P: Use of a submuscular resorbable mesh for correction of severe postpregnancy musculoaponeurotic laxity: An 11-year retrospective study. Plast Reconstr Surg 2008; 121(4): 1240-1248. 